\title{
EDUCATION AND TRAINING SIMBA as an alternative and/or an adjunct to pre-medical work experience during the COVID-19 pandemic
}

\author{
Authors: Nia Evans, ${ }^{\mathrm{A}}$ Meri Davitadze, ${ }^{\mathrm{B}}$ Arjun Narendran, ${ }^{\mathrm{C}}$ Seren Evans, ${ }^{\mathrm{D}}$ Lucretia Thomas, ${ }^{\mathrm{E}}$ Parisha Blaggan, ${ }^{\mathrm{E}}$ \\ Eka Melson ${ }^{F}$ and Punith Kempegowda ${ }^{G}$
}

\begin{abstract}
Work experience is considered as a vital part of an application to medical school and other healthcare-related educational programmes. Gaining clinical work experience via various previously available opportunities from healthcare centres has currently become more challenging and less accessible due to the COVID-19 pandemic-related limitations and resource shortages. In order to provide experience in the healthcare field, we conducted a case study by inviting two secondary school students to participate in Simulation via Instant Messaging -Birmingham Advance (SIMBA) as moderators. Despite no previous clinical knowledge, they found it accessible and to be an excellent alternative to the more traditional types of work experience, which had become unavailable to them. We, therefore, propose SIMBA can act as an alternative and/or an adjunct to work experience for healthcare-related courses.
\end{abstract}

KEYWORDS: work experience, pre-medical school, SIMBA

DOI: $10.7861 /$ fhj.2020-0219

\section{Introduction}

Pre-medical school work experience is considered an essential part of the preparation for studying medicine at UK medical schools. ${ }^{1}$ A prospective doctor is expected to have a comprehensive

Authors: Afoundation year doctor, Royal Glamorgan Hospital, Ynysmaerdy, UK; ${ }^{B}$ endocrinologist, Georgian-American Family Medicine Clinic Medical House, Tbilisi, Georgia; '

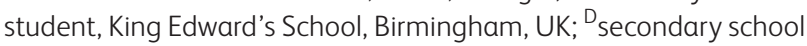
student, St John Baptist High School, Aberdare, UK; ${ }^{\text {}}$ medical student, University of Birmingham Medical School, Birmingham, UK; F foundation year doctor, Ninewells Hospital, Dundee, UK and honorary clinical research fellow, Institute of Metabolism and Systems Research, Birmingham, UK; ${ }^{G}$ Wellcome Trust clinical research fellow, Institute of Metabolism and Systems Research, Birmingham, UK and honorary specialist training registrar in endocrinology, diabetes and general internal medicine, University Hospitals Birmingham NHS Foundation Trust, Birmingham, UK understanding of the qualities required for a career in medicine and to have shown such traits in their previous occupational experiences. ${ }^{2}$ These traits include empathy, teamwork, communication, leadership and problem-solving abilities. ${ }^{3}$

Pre-medical-school work experience, both in clinical medicine and research, provides a more in-depth understanding of being a healthcare professional. Clinical work experience generally involves shadowing healthcare professionals in a medical setting and observing the doctor-patient interaction. On the other hand, research opportunities are facilitated through providing experience in research, preferably with relevance to clinical practice. ${ }^{4}$

Involvement in research is particularly beneficial for pre-medical students as this allows them to form important relationships with mentors who can prepare them for a future career in academia. ${ }^{5}$

The COVID-19 pandemic is having a significant impact on the availability of medical education. ${ }^{6}$ Prospective medical students have undergone difficulties in terms of gaining pre-medical school experience due to stringent infection control actions leading to cancellation of most previously available clinical work experience opportunities. Consequently, diligent applicants have had to seek alternative methods of proving commitment to their chosen course. Therefore, implementing alternative educational programmes that provide clinical work experience to pre-medical students during critical situations (like the COVID-19 pandemic) is essential to assure the progress of studies for future medical students.

\section{What is Simulation via Instant Messaging - Birmingham Advance?}

Simulation via Instant Messaging - Birmingham Advance (SIMBA) is a real-time simulation-based medical training programme where learning is facilitated by WhatsApp and Zoom. SIMBA has proven to be efficient to improve clinicians' confidence in managing various cases in diabetes and endocrinology. ${ }^{7}$

The steps building up to a SIMBA session can be divided into two main phases (Fig 1). In the preparation phase, anonymised transcripts based on real-life cases are prepared. These transcripts reflect the exact journey of a patient with a particular medical condition or disease. Once finalised, these transcripts are critically reviewed and validated by a consultant to ensure the accuracy. Each transcript follows a simple traffic light system of highlighted text. Text highlighted in green is sent to the participant by their 


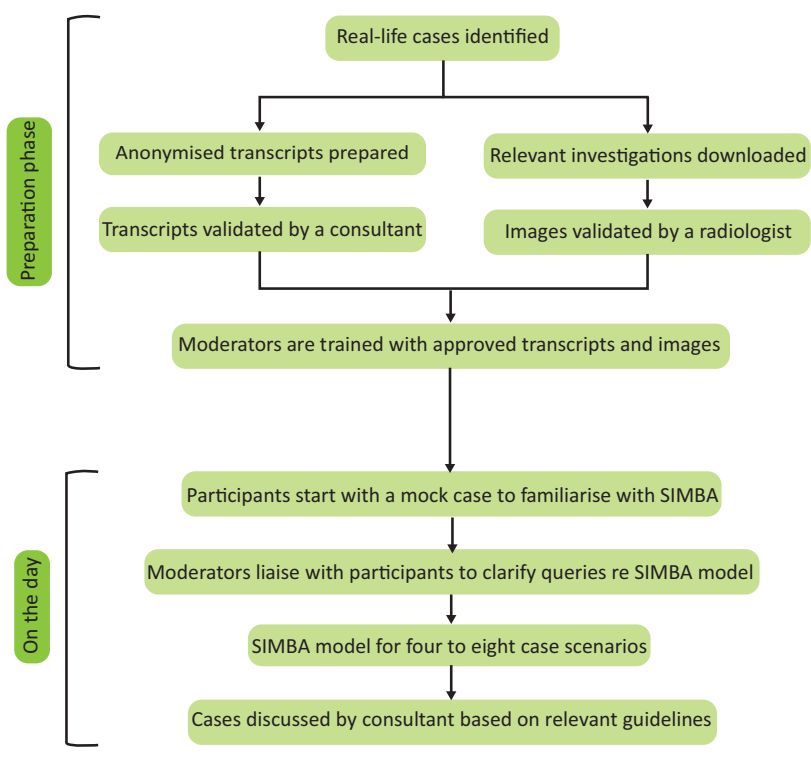

Fig 1. Stages of SIMBA from case preparation to final discussion. SIMBA $=$ Simulation via Instant Messaging - Birmingham Advance.

moderator; this usually either provides the participant requesting a history or asks them to answer a question regarding diagnosis or management. Text highlighted in yellow is instructions for moderators, and text highlighted in red is used by the expert during discussion post-simulation to explain the rationale for diagnosis and management.

The moderators, mainly consisting of medical students, attend training sessions to familiarise themselves with the transcripts. During training sessions, half of the students play the role of a participant and the other half as a moderator. They then swap their role for the next transcript. Five training sessions are organised to facilitate familiarisation with the transcripts. The moderators also have a special session with the session's chair prior to the simulation day where each of these transcripts are discussed in detail, thus helping them understand the simulated cases better.

SIMBA sessions are advertised online, inviting interested participants from anywhere in the world. Registered participants are provided with detailed instructions on how to join the simulation 2 days before the event, including the moderator's WhatsApp number and their unique identifiable number.

On the day of the session, participants join the simulation by messaging their moderators on WhatsApp. Moderators lead the conversation where the participants solve the simulated cases through the standard system of history-taking, examination and investigations to arrive at the diagnosis and propose the relevant management and follow-up plan. To familiarise with the SIMBA model, the first case is run as a trial simulation, after which the moderators liaise with participants to ensure there are no queries or concerns regarding the model.

Following the simulation, participants and moderators join an interactive discussion and question-and-answer session via Zoom where an expert highlights the important learning points from the simulated cases and answers questions raised throughout the session. The participants receive feedback on their performance based on the Global Rating Scale.

\section{Benefits of SIMBA to pre-medical students}

SIMBA offers an opportunity to understand the structure of medical history and terminology, thus preparing pre-medical students for medical school. SIMBA is equally accessible to all students, having a potential to widen access to medical school for secondary school students from disadvantaged socioeconomic backgrounds who find it more difficult to acquire work experience. This is largely due to the relative absence of healthcare professionals within their social network. ${ }^{8}$ Furthermore, SIMBA brings together secondary school students, medical students and junior doctors, encouraging them to contribute to the same purpose. For secondary school students from disadvantaged socio-economic backgrounds, this sort of support and mentorship is invaluable. ${ }^{9}$

Participants require a sufficient foundation of medical knowledge in order to come to a diagnosis and formulate a management plan from the information their moderator gives them. Therefore, it will not be suitable for pre-medical students to be participants. However, observing the simulation will provide limited exposure to the process compared with being directly involved. Because the role of the moderator requires minimal medical knowledge, we feel it is more engaging for aspiring premedical students to be involved in all steps of the SIMBA sessions, as opposed to simply observing.

In order to see if SIMBA could also serve as pre-medical school work experience, two 17-year-old secondary school students were recruited as moderators for our recent SIMBA acute medicine session. Here is their perspective on the programme along with the training lead's experience on working with them.

Student 1's SIMBA experience:

I was first introduced to SIMBA by the moderators' training lead in SIMBA. As I am applying to study optometry in university, I volunteered to moderate for the SIMBA session to gain experience within the healthcare field.

Before the pandemic, I secured placement for work experience in hospitals, care homes and at my local opticians. However, these opportunities were prematurely ended due to COVID-19 restrictions. Under the circumstances, SIMBA was the perfect adjunct to my existing work experience. It was extremely convenient to be part of an international training programme that, although centred in Birmingham in England, I was able to access from home in Wales.

As a school student, initially I found the training process challenging as I felt overwhelmed and lacked confidence to work alongside medical students and junior doctors. Nevertheless, their welcoming and supportive attitude made me feel confident to engage in conversations and ask for help when needed.

On reflection, I would encourage other pre-medical school students interested in medicine to join SIMBA. As a school student you are not overlooked. I was fully involved in every aspect of the simulation and had the independence to make my own decisions while moderating. I was encouraged to take a hands-on approach, directly from those whom I aspire to be. Secondly, SIMBA is not only exclusively beneficial for prospective medical students, but also a valuable experience 
for those considering other healthcare-related courses like myself. On many levels, it provided me with an insight into what a healthcare-associated degree and future career would entail.

Student 2's SIMBA experience:

SIMBA was introduced to me through one of its founders. I am applying to study medicine this year and thought that it would be a great way to boost my application by participating in an innovative teaching model.

While the medical terms used were largely unfamiliar to me, there were certain conditions (such as diabetic ketoacidosis [DKA]) that I had heard before through my other clinical work experience. This prompted extra reading into DKA to learn more about the condition and its aetiology. This kind of interest in specific medical conditions is hard to provoke due to lack of regular exposure to the world of medicine. Therefore, work experience like SIMBA where we can learn about specific medical conditions is extremely helpful.

The most valuable outcome of my participation in SIMBA as a moderator is a better understanding of history-taking and how to evaluate a patient from the time of presentation until diagnosis and management. I can also use my experience as a moderator to communicate efficiently with a wide variety of people and nationalities.

Furthermore, there was a strong element of confidentiality throughout the whole process. I was expected to remain anonymous while being a moderator, and it was heavily reinforced that no personal details should be shared between the moderator and the participant. To me, SIMBA was a better tool for learning about confidentiality, rather than simply observing doctors' interactions with patients in hospitals.

A quality of SIMBA that lends itself to be a good adjunct to pre-medical school work experience is the regularity of the sessions. The programme runs at least one session per month. This sustainability allows applicants to prove their on-going commitment to medicine: a character trait that medical schools are so keen to observe in prospective candidates.

SIMBA training lead's experience:

Due to little previous clinical exposure, the main challenge that the students faced during SIMBA moderation was comprehension of medical terminology. We, at SIMBA, anticipated this and provided an introductory session to teach them the structure of medical history-taking and explain the necessary terminology. The simplicity of the transcripts aided them to quickly grasp what was expected of them.

During the SIMBA session, each student moderated two participants. They were both well prepared for this by having been trained to moderate three participants simultaneously during the training sessions. They were pleasantly surprised to find the pace was slower compared with the training sessions as the participants took time to reply with queries and comprehensive management plans. Some of the participants were international and therefore used different terminology. For example, some used the term 'vital signs' rather than 'observations'. This was challenging for the students having only recently acquainted themselves with the basic medical terminology used in SIMBA transcripts. However, with a core moderator assigned to support them with challenges (such as terminology), both students gave positive feedback on their success in moderating.

\section{Limitations}

While SIMBA can serve as an alternative/adjunct to traditionally accepted clinical work experience, it only introduces pre-medical students to theoretical aspect of medicine. This may limit their ability to demonstrate practical skills of interacting with the hospital team and the patients. On the other hand, prior shadowing of healthcare professionals is useful to fully understand those roles, thus shaping the decision to pursue a career in medicine. Although communication and teamworking is a crucial aspect of SIMBA, it may not match the experience of face-to-face interactions with patients and the multidisciplinary team.

Only two pre-medical school students have moderated for SIMBA to date. We plan to recruit more pre-medical school student moderators via an official online sign-up form in our future sessions. While pre-medical school students find SIMBA to be worthwhile, they found the preparation to be time consuming. We have since adapted our training based on this feedback and reduced the number of moderators' training sessions, which has been better received in our recent sessions.

\section{Conclusion}

SIMBA has previously proved to be an effective learning model to improve clinicians' confidence to manage various clinical cases. Our A-level students' experience suggests that SIMBA may also act as an alternative or an adjunct to work experience for healthcare-related courses for pre-medical school students. This has been even more apparent during the COVID-19 pandemic as other more traditional pathways of work experience have become unavailable. To join SIMBA as a moderator, no prior clinical knowledge is required, just a conscientious character and access to WhatsApp and Zoom.

Any medical or A-level student interested in becoming a SIMBA moderator or, alternatively, any doctor interested in signing up to a SIMBA session as a participant can find further details on the SIMBA simulation website bit.ly/simbasimulation or email the SIMBA team at simbasimulation@gmail.com.

\section{Acknowledgements}

We thank the Institute of Metabolism and Systems Research and University of Birmingham for their continued support for SIMBA. We thank Cai Ying Ng, Dengyi Zhou, Emma Ooi, Issy Allison, Thia Hanania, Wentin Chen and the rest of the SIMBA team for their support with SIMBA. We also thank Dr Behnoush Foroutan Gustafsson, Dr Jonathan W Mueller and Jasline Jaiswal for critically reviewing the manuscript.

\section{References}

1 Park J, Philipp R, Hughes A. Do we value work experience before medical school? Br J Gen Pract 2005;55:242-3.

2 Lin KY, Parnami S, Fuhrel-Forbis A et al. The undergraduate premedical experience in the United States: a critical review. Int J Med Educ 2013:4:26-37. 
3 Marley ], Carman I. Selecting medical students: A case report of the need for change. Med Educ 1999;33:455-9.

4 Davis JM, Anderson MC, Stankevitz KA, Manley AR. Providing premedical students with quality clinical and research experience: The tobacco science scholars program. Wis Med J 2013;112:195-8.

5 Lescak EA, O'Neill KM, Collu GM, Das S. Ten simple rules for providing a meaningful research experience to secondary school students. PLoS Comput Biol 2019;15:e1006920.

6 Ferrel MN, Ryan J]. The impact of COVID-19 on medical education. Cureus 2020;12:e7492.

7 Melson E, Davitadze M, Aftab M et al. Simulation via instant messaging-Birmingham advance (SIMBA) model helped improve clinicians' confidence to manage cases in diabetes and endocrinology. BMC Med Educ 2020;20:274.
8 Brosnan C, Southgate E, Outram S et al. Experiences of medical students who are first in family to attend university. Med Educ 2016;50:842-51.

9 Martin AJ, Beska BJ, Wood G et al. Widening interest, widening participation: Factors influencing school students' aspirations to study medicine. BMC Med Educ 2018;18:117.

Address for correspondence: Dr Punith Kempegowda, Institute of Metabolism and Systems Research, University of Birmingham, IBR Tower, Level 2, College of Medical and Dental Sciences, Edgbaston, Birmingham B15 2TT, UK.

Email: p.kempegowda@bham.ac.uk

Twitter: @drpunith 